University of New Hampshire

University of New Hampshire Scholars' Repository

6-2011

\title{
Remote characterization of seafloor adjacent to shipwrecks using mosaicking and analysis of backscatter response
}

\author{
Giuseppe Masetti \\ University of New Hampshire, Durham, giuseppe.masetti@unh.edu \\ Roberto Sacile \\ Università degli Studi di Genova \\ Andrea Trucco \\ Università degli Studi di Genova
}

Follow this and additional works at: https://scholars.unh.edu/ccom

Part of the Oceanography and Atmospheric Sciences and Meteorology Commons

\section{Recommended Citation}

G. Masetti, R. Sacile, and A. Trucco, "Remote characterization of seafloor adjacent to shipwrecks using mosaicking and analysis of backscatter response," Italian Journal of Remote Sensing, pp. 77-92, Jun. 2011.

This Journal Article is brought to you for free and open access by the Center for Coastal and Ocean Mapping at University of New Hampshire Scholars' Repository. It has been accepted for inclusion in Center for Coastal and Ocean Mapping by an authorized administrator of University of New Hampshire Scholars' Repository. For more information, please contact Scholarly.Communication@unh.edu. 


\title{
Remote characterization of seafloor adjacent to shipwrecks using mosaicking and analysis of backscatter response
}

\author{
Giuseppe Masetti ${ }^{1}$, Roberto Sacile ${ }^{2}$ and Andrea Trucco ${ }^{3}$ \\ ${ }^{1}$ Istituto Idrografico della Marina, Passo Osservatorio, 4 - Cap 16134 Genova, Italy \\ ${ }^{2}$ Università degli Studi di Genova - DIST, Via Opera Pia 13 - Cap 16145 Genova, Italy \\ ${ }^{3}$ Università degli Studi di Genova - DIBE, Via Opera Pia 11A - Cap 16145 Genova, Italy \\ E-mail: giuseppemasetti@yahoo.it
}

\begin{abstract}
The paper's aim is to evaluate mosaicking and analysis of backscatter angular responses as adequate techniques to quickly characterize the seafloor adjacent to shipwrecks, extending the results of a limited number of grabs. Both techniques have been applied to the casestudy of the VLCC Haven shipwreck site, applying the approach known as Geocoder among the available methods. From these results, the development of the research activities will attempt to improve techniques and to generalize a methodological approach for the analysis of backscatter coming from an area of seafloor with the presence of one or more anthropic objects.
\end{abstract}

Keywords: backscatter, mosaic, multibeam, seafloor classification, shipwreck.

\section{Introduction}

An acoustic wave propagating in water meets a series of obstacles either in the water column itself (e.g., plankton, air bubbles, fish shoals) or at the boundary interfaces of the medium (seabed and surface of the sea). After these collisions, a part of the transmitted energy comes back to the sonar system. In the case of a monostatic configuration (transmitter and receiver located at the same point), this energy is called backscatter.

As a function of the available system typology and the related purpose of utilization, the energetic components of an echo are then either desirable (if the obstacle is the target wanted) or not (reverberation jamming the useful signal). In both cases, understanding their properties is essential to the functioning of the acoustic system because the echoes need to be received in the best conditions, or because they need to be reduced or filtered out as much as possible [Lurton, 2002].

Depending on the purpose of the user, there are many different types of information that is possible to extract from the seafloor acoustic data including physical (i.e. porosity), acoustic (i.e. attenuation), geotechnical (i.e. elastic and shear strength moduli) properties, and also the general morphology (i.e. relief) at different scales. In the absence of a specific task, a seafloor characterization system aimed at extracting information about sediment texture probably is most generally useful. In fact, the grain size is important for a number of engineering, environmental, and military applications [Mayer, 2011]. For the particular case of the environmental risk assessment of shipwrecks, this information is useful for many scopes (i.e. what intervention 
adopts to minimize the environmental impact, monitoring of oil patches).

The use of the multibeam echosounders (MBES) started in the seventies for bathymetric purposes. But only in the last two decades their potentialities for seafloor characterization from the reflected energy measurements have emerged [De Moustier, 1991]. In the recent years, a certain number of different approaches have been developed to process the MBES reflectivity data in order to map discrete geographical areas of the seafloor with the same acoustic signature. This process is generally called acoustic segmentation [Preston et al., 2004].

In the work presented in this paper, we analyze the backscatter coming from the seabed acquired by a MBES - to mainly remotely identify the sediment type of an area adjacent to an anthropic object (i.e. the shipwreck of the VLCC Haven). Among the various available ones, the approach called Geocoder is applied [Fonseca and Calder, 2007; Fonseca et al., 2009]. The deducted results are ground-truthed by grabbing.

The expected results of this work are to evaluate the advantages of the Geocoder approach to quickly and entirely identify - with the help of a limited number of seafloor samples - the sediment type of seafloor areas characterized by the presence of shipwrecks.

\section{Backscatter, roughness and angle of incidence}

An acoustic wave meeting an interface between two mediums produces - in function of the angle of incidence, the different acoustic impedances (products of sediment density and sound speed), and other parameters that are characteristics of each medium - a set of waves refracted in the second medium and another set reflected and diffused (scattered) in the first one.

The ratio between the acoustic intensity coming back from the target in direction of the source and the intensity incident on the same obstacle is called target strength (TS). This ratio is related to the physical nature of the obstacle, to the structure (internal and external) and to the characteristics of the incident signal (angle and frequency).

During the propagation, the intensity of the transmitted acoustic wave (source level, $S L$ ) is subject to transmission loss (TL) mainly due to geometrical spreading and medium absorption.

In the specific case of the seabed, the target strength mentioned before can be determined using the backscattering cross-section which can be decomposed into two parts: the dimension of the seabed actually insonified by the acoustic system $\left(A_{s}\right)$ and the corresponding backscattering strength for unit of surface $\left(B S_{s}\right)$.

So the level in decibel of the backscattering signal (echo level, $E L$ ) can be estimated using the monostatic active sonar equation [Kinsler at al., 2000]:

$$
E L=S L-2 T L+T S=S L-2 T L+10 \log _{10} A_{S}+B_{S}[1]
$$

The interactive process is influenced by the local characteristics of the seabed, which - as a function of the frequency and of the angle of the incident acoustic wave - can be more or less represented as an ideal plan surface. The irregularities of the surface shed the acoustic energy in all the directions (Fig. 1): one part, called coherent, is reflected without 
deformations - except the transmission loss - in a direction specular to the incidence; whereas, the remaining part is scattered all around the space, including the direction of the source (backscatter). The relation between the two over mentioned parts - specular and scattered - is mainly a function of the surface roughness.

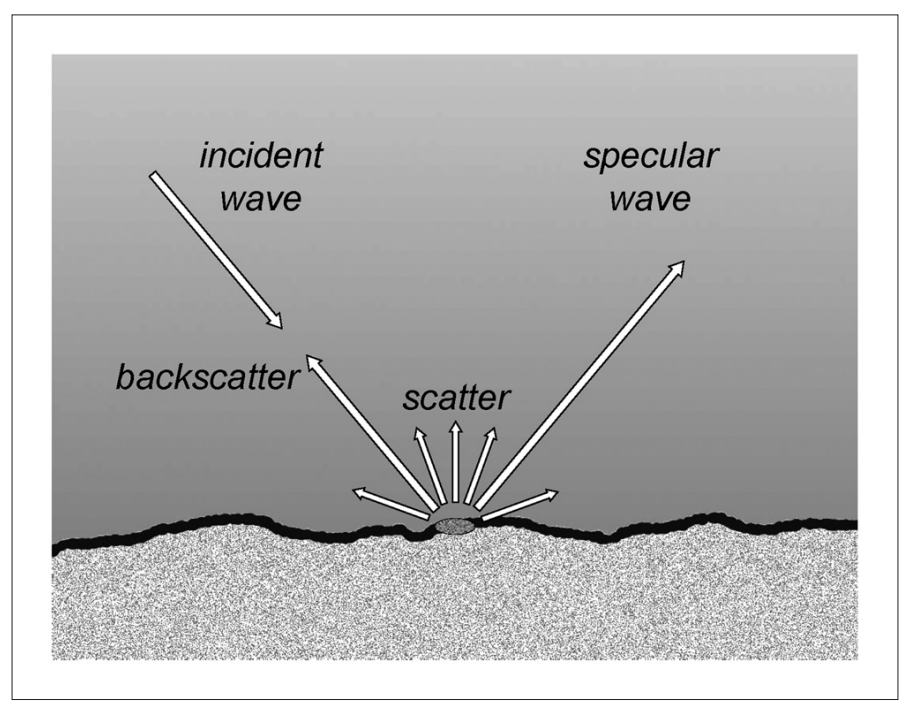

Figure 1 - Reflection (coherent part) and scattering of an acoustic wave incident a rough surface.

The roughness of the seabed - depending by the geological (i.e. rock, mud, ...) or biological (living organism, shell, ...) origin - covers a wide scale of sizes (from a few millimeters to meters). Furthermore, there are some scales - i.e., ripples of some centimeters present on the topography of a sandy seabed - which produce different physical processes at the same time. Applying the Fourier transform to the seabed relief, it is possible to quantify the energetic distribution in different spatial harmonic components, each one defined by a spatial wavelength $(\Lambda)$ and a wave number $(K=2 \pi$ $/ \Lambda)$. In the case of a surface of random roughness, the power spectrum $\left(S_{K}\right)$ tends to decrease with wave number, as larger amplitudes are associated with longer wavelengths (Fig. 2). For a given seafloor area, the variance $h^{2}$ of relief amplitude can be estimated by the integral of its spatial spectrum over all components [Lurton, 2002]:

$$
h^{2}=\int S_{K} d K \quad[2]
$$

Beyond the surface roughness, the scattered part of the acoustic signal is tightly influenced by the angle of incidence. For angles of incidence close to the normal, the incident wave is reflected by the facets oriented to work as a mirror. This way corresponds to the maximum level of measured backscatter intensity and it is called 
facet reflection. At oblique angles of incidence, the backscatter comes back as a product of a series of little contributors (scatterers), variously distributed in function of the local roughness. This process occurs in the so-called Bragg scattering domain.

A composition of the two over mentioned regimes (facets reflection and Bragg scattering) - in function of the local roughness - can be used to realize a simple theoretical model for the scattering of an acoustic wave on a rough surface (Fig. 3).

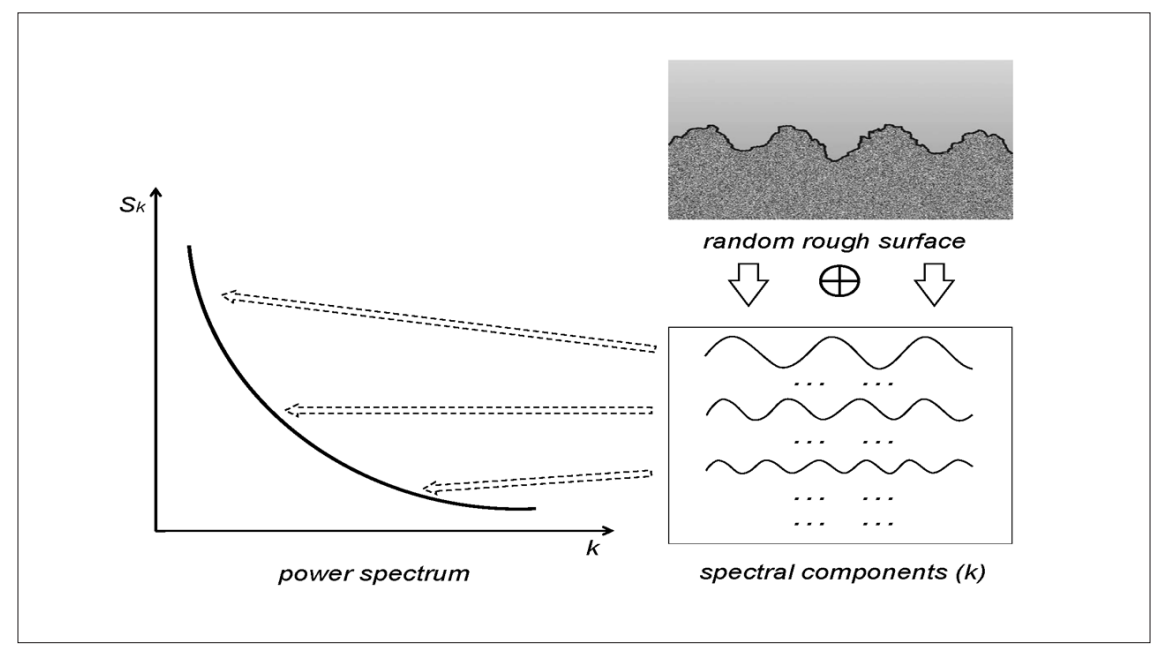

Figure 2 - Spatial spectrum of a random rough surface through Fourier transform of the relief.

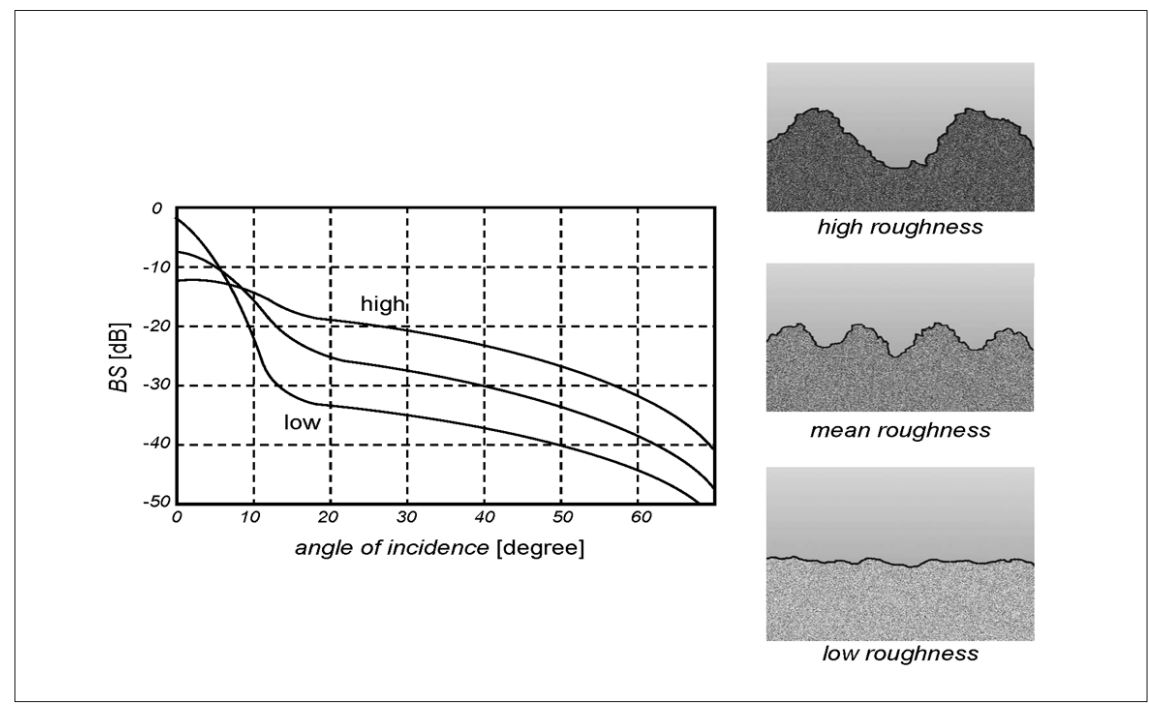

Figure 3 - Simple model of backscattering strength response of the seafloor in function of the angle of incidence and the microscale roughness. 


\section{Acoustic response of the seabed}

The seabed mainly acts as a rough surface which spreads the incident acoustic wave. Among the energy scattered, the backscatter represents the source of information used by the acoustic seafloor mapping systems.

Part of the incident intensity can penetrate into the seabed due to the little contrast of impedance between water and sediments. At the high frequency usually used by shallow-water MBES, the bottom penetration is small and the interaction is bounded to the surface (reflection from a non-plane interface), with possible complications related to the stratified or heterogeneous nature of the sediment.

Heterogeneities can have various natures, but they are mainly represented by air bubbles entrapped in the sediment. Their response is usually modeled by a random distribution of scatterers whose contributes interferes with each other.

In literature there are different parameters to describe the variety of sediment types. The most common parameters representative of the sediment hardness are: the main grain size in Phi units $\left(M_{\varphi}\right)$; the porosity $(n)$; the density $(\rho)$; the relative compressional velocity $\left(c_{r}\right)$ referenced to the speed of sound in water; the absolute compressional velocity $(c)$ for a seawater value of $1,500 \mathrm{~m} / \mathrm{s}$; the reflection coefficient at normal incidence $\left(V_{0^{\circ}}\right)$; the compressional wave absorption coefficient $(\alpha)$; the absolute shear wave velocity $\left(c_{s}\right)$; etc. Whilst, parameters representative of the sediment roughness may be: the roughness spectral strength $\left(\Omega_{0}\right)$, the standard deviation of the roughness along a unit distance $(h)$, and the roughness slope standard deviation $(\delta)$.

There is an obvious correlation between the sediment hardness and its roughness. For example, the roughness parameters globally increase with the acoustic impedance and, then, with the mean grain size of the sediment.

At frequencies used by acoustic seafloor mapping systems - from tens to hundreds of $\mathrm{kHz}$ - the backscatter coming from the seabed can be separated into a part scattered by the interface relief (either as facets reflection close to the nadir or by the scatterers due to local roughness at grazing angle) and another part which penetrates the sediment and is reflected back by volume heterogeneities (Fig. 4). This last process can also become predominant at oblique angles of incidence.

\section{MBES imaging and remote characterization}

The principle of sonar imaging for a MBES is mostly the same of a sidescan sonar system (SSS): the signal backscattered is recorded as a function of time and its instantaneous intensity varies with the roughness and so with the nature of the insonified seabed strip swept by the signal.

But there are differences which make the process more complicated: with MBES data, time signal is available only after the beamforming and the sonar image is performed on the basis of a digital terrain model (DTM) created by bathymetric measurements; the central point of a beam is positioned on the swath and the image pixels are distributed around it until the boundary with the neighbor beam is reached. The sonar image so obtained has a geometrical distortion more reduced than a SSS imaging, which uses only the oblique-distance measurement and supposes a plane surface. At the same time, the presence of co-located bathymetry and reflectivity information in the MBES data permits to apply at the backscatter values more accurate corrections due to the effects of footprint size and local slope. 
However, using systems at comparable frequencies, the quality of MBES imagery is generally less satisfying than SSS products. This is due to different issues: the alongtrack resolution is usually less fine; the directivity diagrams, although they improve the SNR, are prone to modulate the amplitude of the data collected (i.e., the resulting image without appropriate corrections is striated at constant angles parallel to the ship's track); the angle of incidence of a MBES - usually hull mounted - is less grazing than for a SSS towed close to the seafloor. In particular, this last issue makes the detection of intensity contrast for micro-relief facets difficult.

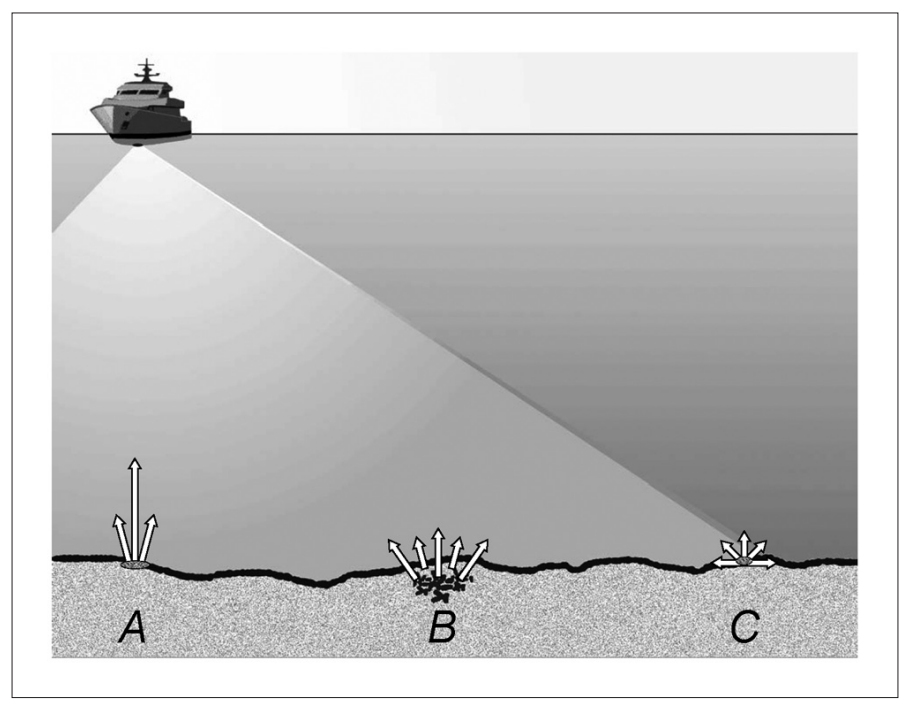

Figure 4 - Processes involved in the backscatter response: A) facets reflection, B) volume heterogeneities and C) scatterers.

For all the above mentioned arguments, it is easy to understand why the quality of a MBES image is usually lower than one obtained by SSS data. So MBES imaging should be more properly assimilated to measurement of average reflectivity and used to map variations in seabed types.

As for many common applications (navigation, dredging, fishery, ...) only a restricted identification of the seabed type - i.e. using an around ten classes - is required, this objective can simply be obtained by the analysis of the measured backscatter. However, none of the geological (granulometry, mineral constituents, ...) and geotechnical (density, compression modulus, ...) characteristics of the sediment can be directly identified by MBES reflectivity.

It is also possible trying to extract some geological and geotechnical sediment parameters by comparison with a model. This approach - only proven under simplifying assumptions - has a limited validity for the number of parameters to obtain in comparison of the small number of information that can be extracted from the backscattered acoustic signal.

Two main approaches coexist to characterize the seabed with MBES. The first - surely 
more ambitious - aims at solving the "inverse problem": to extract the characteristics of the sediment from the acoustic data available. This implies large modeling efforts to correlate acoustics data observed experimentally and the geological characteristics, in particular for the presence of porous media, living organisms, variously layered sediments, etc.

This approach, theoretical reachable, is too highly conditional - a large number of input parameters and very few observations - and sometimes hard to ground-truth, i.e. in deepwater [Lurton, 2002]. However, an increasing number of mathematical models has been developed to obtain a better physical understanding of backscatter coming back from the seabed [Mulhearn, 2000]. There are models that consider backscatter versus grazing angle, i.e. Stockhausen [1963], Crowther [1983], Stanton [1984], Jackson et al. [1986], Boyle and Chotiros [1995], and other which consider its behavior versus time from a pulse transmission, i.e. Bergem et al. [1999], Clarke et al. [1988].

The second approach - definable pragmatic - categorizes useful classifying parameters among the data acquired by the MBES. Also if usually deprived of any direct physical significance, these parameters are provided of sufficient discriminatory power. The MBES data are calibrated in function of these parameters, over a certain number of validated configurations. If there is a new case, it can be identified by comparing them with the existing database.

Despite this approach can appear restrictive, the largest part of the practical applications available nowadays are based on it. For instance, RoxAnn [Murphy et al., 1995] and QTCView [Collins et al., 1996], ones of the first commercial systems in this field, work on the output of a standard ship's echosounder to analyze in various way the acoustic returns from the seafloor. These systems are not seafloor classifiers, but rather segmentation systems that divide the seafloor into regions of consistent acoustic return type [Mayer, 2011]. The results obtained - empirical and site specific - require a certain number of sediment samples to correlate acoustic answers and seabed parameters [Hamilton et al., 1999].

Recent approaches to the problem of the acoustic segmentation are mainly based on: the texture analysis applied to backscatter mosaic [Preston et al., 2004; Preston, 2009], the extraction of features based on the relationship between the reflectivity and the angle of incidence [Hughes Clarke, 1994; Fonseca and Calder, 2007; Fonseca and Mayer, 2007; Parnum et al., 2007], the analysis of entire backscatter curves [Canepa and Pace, 2000; Hamilton and Parnum, 2011].

\section{Issues linked to the backscatter mosaicking}

A backscatter mosaic image is a grid of square pixels, each one representing an area of $m x$ $m$ square meters on the seabed [Augustin et al., 1994]. It is built by grouping and averaging individual backscatter intensity - at different angles of incidence and from different acoustic swaths - into pixels values representing a normalized amplitude and the original angular information removed. At the same time, as a consequence of the mosaic resolution chosen, some pixels may contain interpolated value from the surrounding measured reflectivity values.

In the mosaicking process, there are two main obstacles: MBES does not log directly the absolute value of backscatter, but relative magnitude whose calculation is not clearly defined in the technical documentation of the system manufacturer; the need to remove the backscatter angular response, which represents how the backscatter strength changes with the angle of incidence.

The removal of the angular variations of the backscatter, due to different angular response, is 
an essential operation to create a consistent mosaic - without angular variation along the swath - for an area of homogeneous seafloor.

This result is difficult to obtain because the angular response is an intrinsic characteristic of the seafloor. Thus, we need to know some information about the seabed type before to create the relative backscatter mosaic. This prerequisite is not properly practical as the primary aim of an acoustic mosaic is proper to obtain some indications around the seafloor nature [Fonseca and Calder, 2007].

\section{Analysis of the backscatter angular response}

The variation along-swath of the backscatter intensity linked to the angle of incidence - one of the two problems for a correct mosaicking - represents, at the same time, the main source of information in various methods of remote seafloor characterization. In practice, by the analysis of the backscatter angular response, it is possible to estimate important acoustic and physical properties as grain size, impedance, attenuation and roughness of the superficial sediments.

In this technique, the signal level for a given beam, sometimes averaged on a series of consecutive pulses, is corrected for some terms of the sonar equation [1] to obtain the target strength. This is then corrected for the swath footprint in function of the geometry, of the beam amplitude and of the signal time duration. So the unit backscattering strength obtained - corrected by the refraction for the sound velocity profile and by the bathymetry extracted by the digital seabed model - is assumed to be only function of the angle of effective incidence on the seafloor and the sedimentary facies.

However, the information obtained is enough coarse as it is possible to fall into ambiguities due to different seabed types with similar acoustic responses at the same angles. Moreover, there are two additional sources of difficulties in the application of the above method: inaccurate measurements of backscatter strength and not uniform sediment types of seafloor across the swath [Fonseca et al., 2009].

\section{The case-study of the VLCC Haven}

The unfortunately famous VLCC Haven shipwreck - one of the biggest of the Mediterranean with its 344 meters of length overall - has been chosen as a case-study to apply the mosaicking and the analysis of the backscatter angular response. The main body lies on a muddy seafloor off the coast of Arenzano (Genoa) at a depth of about 75 meters (Fig. 5 and Fig. 6), whilst a part of the bow with the bulb is at more than 500 meters of depth.

The sinking - dating back to April $14^{\text {th }}, 1991$ - was due to a fire caused by a violent explosion abreast of the forward tank. After the sinking, the combusted and semi-combusted residues were removed from the seashore and the seabed as far as the 10-meters isobath, and were pumped from the shipwreck tanks and premises. The residues present on the seabed at a depth beyond 10 meters have been left to environmental auto-decomposition processes.

In 1998, at the end of a dispute lasting about eight years, the Italian Prime Minister solved the dispute concerning compensation for the sinking related damages, with an out-of-court settlement. Part of the damage compensation was employed to perform both decontamination operations on the shipwreck and interventions of environmental restoration of the sea and coast area, which were most damaged by the accident's harmful effects. The shipwreck decontamination operations formally ended on June $12^{\text {th }}, 2008$. 


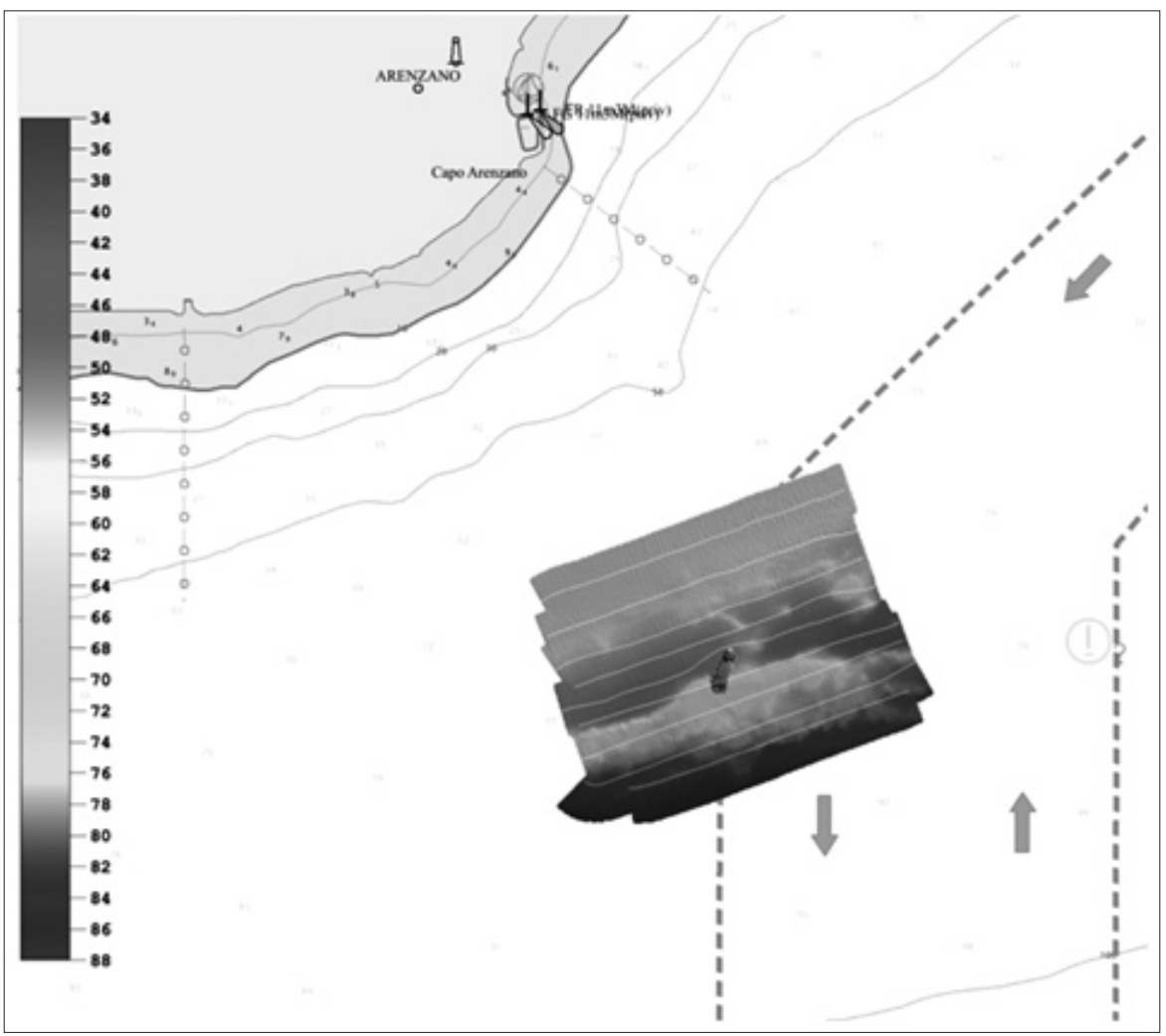

Figure 5 - Bathymetric map (in meters) of the case-study area.

From the environmental point of view, the decontamination operation of the Haven represents an important pilot experience at the international level because of both the location of the hydrocarbon tanks and the shipwreck position, lying on shallow seabed a few nautical miles from a tourist coastal zone [Masetti and Orsini, 2009].

The acoustic data of this case-study were recorded using the MBES Kongsberg Simrad EM300, hull mounted at the Aretusa ship, hydrographic vessel of the Italian Navy [Masetti et al., 2010]. The tool Mosaic Editor - based on the Geocoder engine - of the hydrographic data processing software Caris HIPS\&SIPS 7 was used to create mosaics and analyze the sediment properties of the acoustic image obtained. This choice was mainly due to the array of detailed backscatter corrections and accurately modeled seafloor characterization algorithms of the Geocoder approach.

The workflow, started with the import of the acquisition lines, was developed with the application of tide corrections and the editing to remove any spikes or bad data through the editors for navigation, attitude and bathymetry. After this cleaning, a step common to the bathymetric processing, the Geocoder engine algorithms of backscatter correction were applied to the data [Fonseca and Calder, 2005; Mayer et al., 2007].

The backscatter information are collected by this Kongsberg MBES as reflectivity (backscattering strength) for each beam and seabed image reflectivity (amplitude) for each of the contributor samples at the bottom detection of a beam. The data can be 
used for the seafloor characterization, but require some post-processing corrections to increase their accuracy and remove the distortion of the real-time Time Variable Gain (TVG) model applied during the acquisition [Hammerstad, 2000].

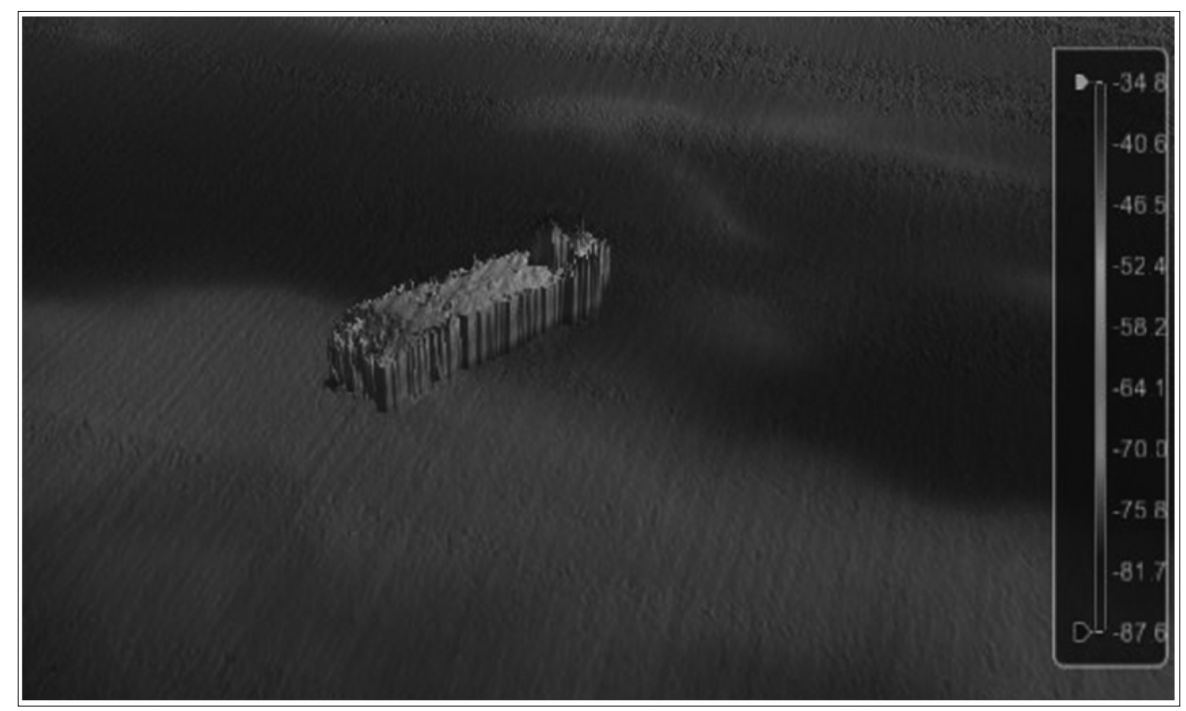

Figure 6-3D view of the shipwreck based on the bathymetric data acquired.

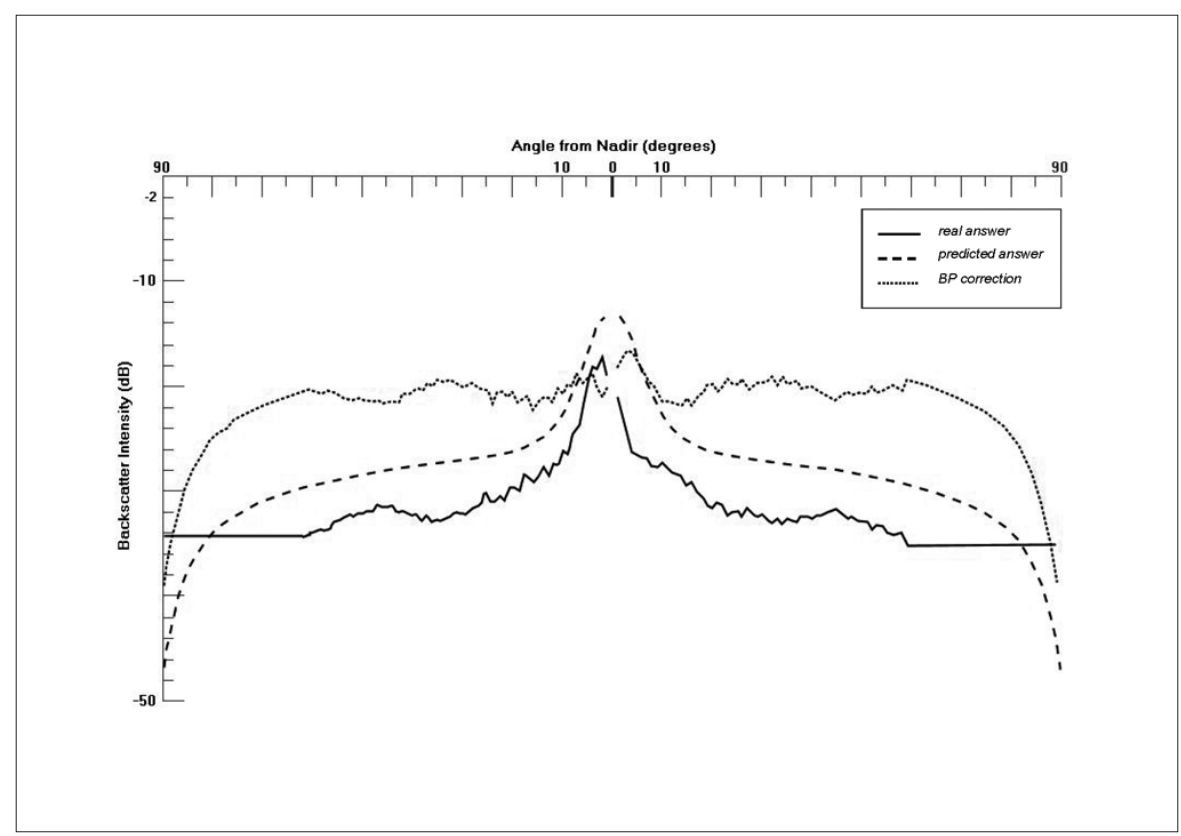

Figure 7 - Beam pattern window showing the real answer of the transducer, the predicted one on the basis of the MBES frequency and sediment type, the resulting correction to apply to remove the transducer artifacts. 
The Kongsberg seabed image data are not corrected for beam pattern variations in the receiver beams. Thus, the beam pattern correction has been applied to remove angular artifacts due to the transducer. This correction is based on a beam pattern file generated by the user to identify and remove this effect (Fig. 7). To obtain a good result, the file has been created on a flat, homogeneous and target-free area characterized by a well known sediment type. This file has been applied to all the survey data to correct them uniformly, obtaining a better acoustic image.

The processed imaging data were stored as Georeferenced Backscatter Raster (GeoBaR). GeoBaRs use BASE Surface technology and share in all of the benefits of that technology such as data caching, which helps provide scalability [MacDonald and Collins, 2008]. Using these GeoBaRs, a preliminary mosaic was created and, after the application of proper supervised adjustments (i.e. contrast, brightness) the final product was realized (Fig. 8).

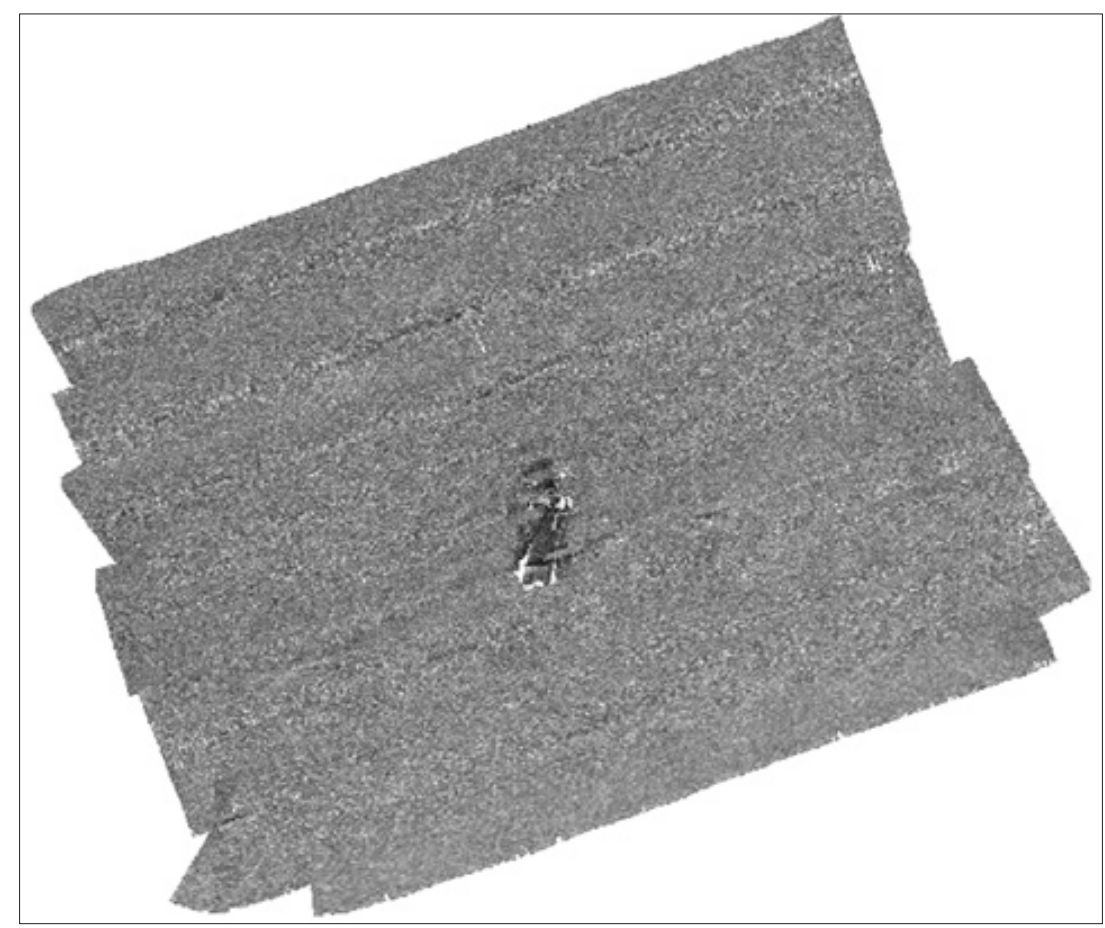

Figure 8 - Backscatter mosaic of the area adjacent to the VLCC Haven shipwreck.

It is now possible to spatially identify mosaic regions with similar acoustic properties - called "acoustic themes" - defined using conventional, subjective and by-eye interpretation. The same method has been proven to be effective for SSS backscatter data, particularly in regions with sharp demarcations between neighboring seabed types [Brown, 2004].

From the analysis of the obtained mosaic, the uniformity of the reflectivity emerges and, 
on the basis of the mentioned acoustic themes assumption, it could be possible to deduct the uniformity of the seabed sediment around the shipwreck object of the investigation. However, this assumption is recognized to be less effective for seafloor with a high level of seabed heterogeneity or a gradual change in the seabed characteristics because the backscatter behavior doesn't present clear demarcations [Brown, 2008].

At the same time, a certain number of artifacts - i.e., corresponding at the normal incidence of some acquisition lines in the northern part of the survey area - are present in the final product (Fig. 9 left); whilst the area closer to the shipwreck is of difficult visual interpretation (Fig. 9 right).

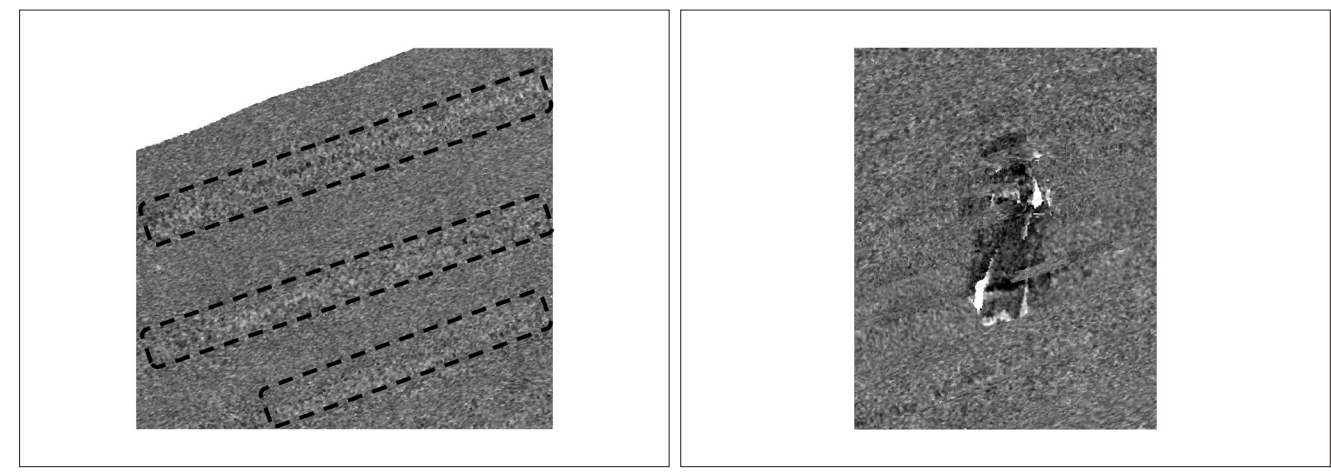

Figure 9-Artifacts present at normal incidence in some acquisition lines in the northern part of the backscatter mosaic (left); zoom on the shipwreck site (right).

An objective automated classification of the acoustic data based on the backscatter signal were conducted to avoid uncertainty or low confidence in the acoustic themes. This classification is based on a series of parameters calculated from the stacking of consecutive time series over a spatial scale that approximates half of the swath width (a stack of $20-30$ consecutive sonar pings). Thus, each stacked angular response defines two distinct seafloor patches (one for the port side and another for the starboard side). These sediment analysis capabilities are one of the most innovative aspects of the Geocoder approach. It is made possible by the accurate removal of acquisition artifacts found in the source data and derived from years of published research in this field. The Geocoder sediment analysis engine - based on the ARA model [Fonseca and Mayer, 2007] and realized in SIPS with the name of Sediment Analysis Tool (SAT) - has been applied to the corrected imaging data. The results returned (i.e., sandy mud and muddy sand) are largely common for the entire area, showing the same homogeneity obtained by the visual interpretation (Fig. 10). This analysis, as in the case of the visual interpretation of the mosaic (Fig. 9 right), has also given results of difficult interpretation and high variability for the area closer to the shipwreck.

Summarizing, the described mosaicking technique, requiring the removal of information linked to the angular response, reduces the ability to make a quantitative seabed characterization on the obtained mosaic and it presents a series of artifacts. Whilst, the analysis of angular responses only permits to characterize the seafloor, but 
with a spatial resolution limited to the swath width of the sonar. A combined approach could improve the spatial resolution of the angular response analysis and could produce mosaics more homogeneous, with fewer along-track and across-track artifacts [Fonseca and Calder, 2007]. The quality of the backscatter analysis and mosaicking results has been directly verified trough the execution of a limited number of seabed samples trough grabs (Fig. 11). Due to the target of this work, limited to the sediment type classification, these samples have been only visual inspected to define the mean grain size.

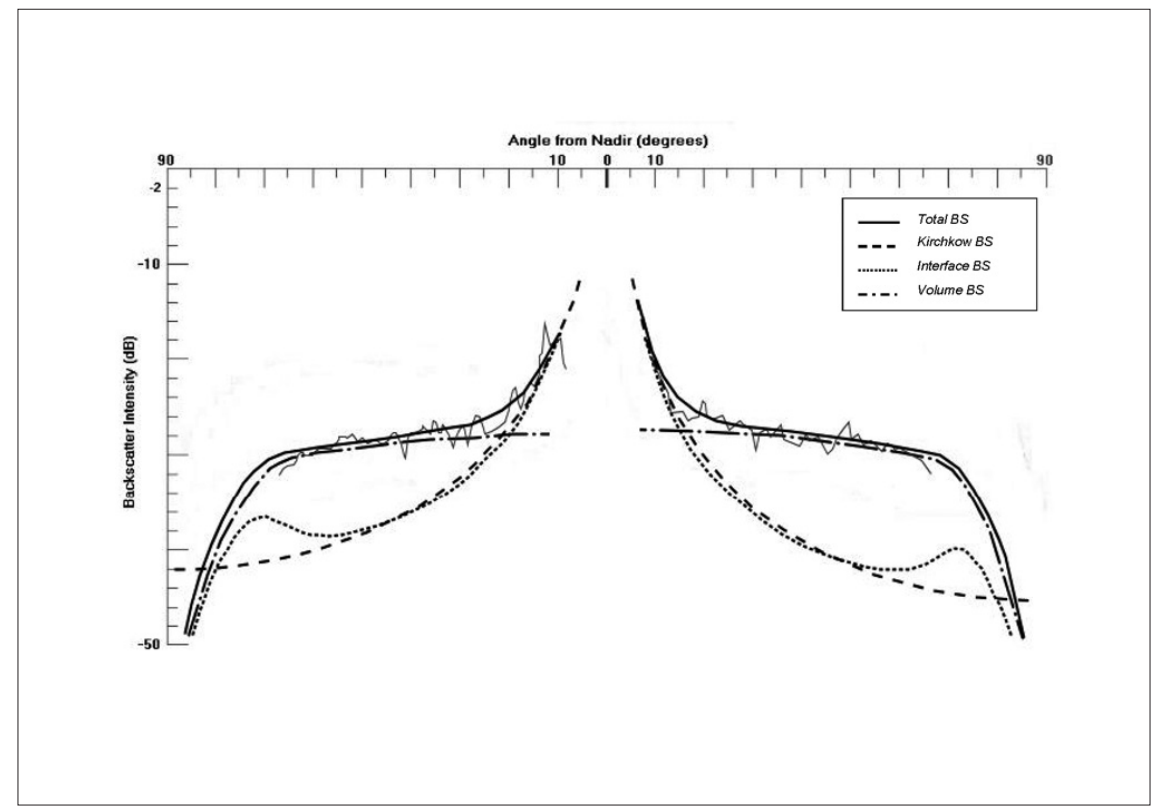

Figure 10 - Sediment Analysis Window: the real backscatter answer is modeled as the product of interface, volume and Kirchoff backscatter components.
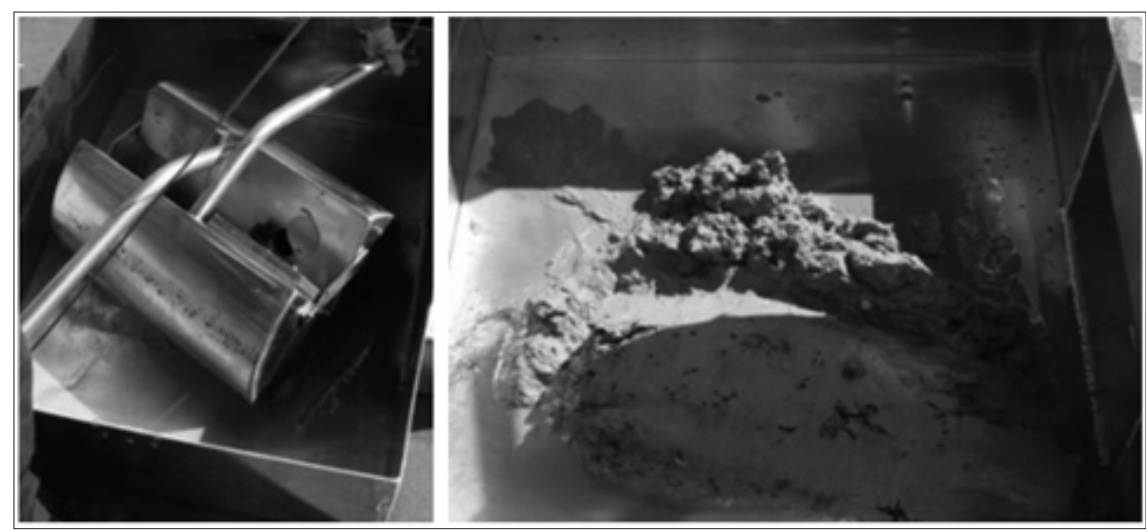

Figure 11 - Seabed sampling trough grabs. 


\section{Conclusions}

The backscatter mosaicking and analysis processes applied to the seafloor adjacent to the VLCC Haven shipwreck have allowed for quickly characterization of the area -2 hours for the MBES acquisition and the sampling operation, less than 1 hour for the data processing, both bathymetry and seafloor classification - extending the results of a limited number of grab samples to the remaining part of the survey with a good level of confidence.

The content of this paper represents a preliminary report, coming from the first part of a University of Genoa Ph.D. research project entitled "Georeferencing and Environmental Risks Monitoring of Shipwrecks using MBES data". From these results, the development of the research activities will attempt to improve techniques and to generalize a methodological approach for the analysis of backscatter coming from an area of seafloor characterized by the presence of one or more anthropic objects.

At the same time, it will attempt to define uniform criteria to collect the information coming from MBES data into a modern shipwrecks' GeoDB. In fact, only the standardization of databases allows the accessibility and comparability for various States [ICRAM CEDRE, 2007]. This characteristic should be a fundamental element for a wreck identification program - in particular for a closed basin as the Mediterranean - to ensure permanent monitoring and continuous updating of the shipwreck sites to prevent pollution effects (i.e., spillage of oil from the tanks).

The main research outcome could be the possibility to use MBES data as a low-cost method to monitor conditions of the large number of shipwreck presents in oceans. Furthermore, this research could give an important contribution to the creation of a Decision Support System (DSS) - based on a large geo-database with vessel, cargo and sink event information - used to define the criteria and the priorities for decontamination operations related to potential polluting shipwrecks.

\section{Acknowledgments}

The authors wish to thank the Cpt. Carlo Marchi and all the crew of the Italian Navy's Hydrographic Ship "Aretusa" for the support during the acquisition of the acoustic data and the ground thruthing operations. The methods and guidelines presented in this paper do not necessarily represent the policy of the Istituto Idrografico della Marina.

\section{References}

Augustin J.M., Edy C., Savoye B., Le Drezen E. (1994) - Sonar mosaic computation from multibeam echosounder, Proc. IEEE Oceans '94, Brest (France), II: 433-438.

Bergem O., Pouliquen E., Canepa G., Pace N.G. (1999) - Time-evolution modeling of seafloor scatter. J. Acoust. Soc. Am., 105: 3136-3141. doi:10.1121/1.424645

Boyle F.A., Chotiros N.P. (1995) - A model for high-frequency backscatter from gas bubbles in sandy sediments at shallow grazing angles. J. Acoust. Soc. Am., 98: 531-541. doi:10.1121/1.413645

Brown C.J., Hewer A.J., Limpenny D.S., Cooper K.M., Rees H.L., Meadows W.J. (2004) - Mapping seabed biotopes using sidescan sonar in regions of heterogeneous substrata: case study east of the Isle of Wight, English Channel. Underwater Technology, 26: 27-36. doi:10.3723/17560540478310155

Brown C.J., Collier J.S. (2008) - Mapping benthic habitat in regions of gradational substrata: 
an automated approach utilizing geophysical, geological and biological relationships. Estuarine Coast and Shelf Science, 78: 203-14. doi:10.1016/j.ecss.2007.11.026

Canepa G., Pace N.G. (2000) - Seafloor segmentation from multibeam bathymetric sonar. Proceedings of the $5^{\text {th }}$ European Conference on Underwater Acoustics, Lyon (France), p. 361-366.

Clarke T.L., Proni J.R., Seem D.A., Tsai J.J. (1988) - Joint CGS-AOML Acoustical Bottom EchoFormation Research I: Literature Search and Initial Modelling Results. NOAA Technical Memorandum ERL AOML-66.

Collins W., Gregory R., Anderson J. (1996) - A Digital Approach to Seabed Classification. Sea Technology, 37: 83-87.

Crowther P.A. (1983) - Some statistics of the sea-bed and scattering therefrom. In Pace N.G. (editor) - Acoustics and the Sea-Bed. Bath University Press, Bath (UK).

De Moustier C. (1991) - Angular dependence of $12 \mathrm{kHz}$ sea-floor acoustic backscatter. JASA, 90 (1): 522-531.

Fonseca L., Brown C., Calder B., Mayer L., Rzhanov Y. (2009) - Angular range analysis of acoustic themes from Stanton Banks Ireland: A link between visual interpretation and multibeam echosounder angular signature. Applied Acoustics, 70: 1298-1304. doi:10.1016/j.apacoust.2008.09.008

Fonseca L., Calder B.(2005)-Geocoder: An Efficient Backscatter Map Constructor. Proceedings of the U.S. Hydrographic Conference 2005, San Diego (CA).

Fonseca L., Calder B. (2007) - Clustering Acoustic Backscatter in the Angular Response Space. Proceedings of the U.S. Hydrographic Conference 2007, Norfolk (VA).

Fonseca L., Mayer L. (2007) - Remote estimation of surficial seafloor properties through the application of Angular Range Analysis to multibeam sonar data. Marine Geophysical Researches, 28 (2): 119-126. doi:10.1007/s11001-007-9019-4

Hamilton L.J., Mulhearn P.J., Poeckert R. (1999) - Comparison of RoxAnn and QTC-View acoustic bottom classification system performance for the Cairns area, Great Barrier Reef Australia. Continental Shelf Res., 19: 1577-1597. doi:10.1016/S0278-4343(99)00020-5

Hamilton L.J., Parnum I. (2011) - Acoustic seabed segmentation from direct statistical clustering of entire sonar backscatter curves. Continental Shelf Research, 31: 138-148. doi:10.1016/j.csr.2010.12.002

Hammerstad E. (2000) - Backscattering and Seabed Image Reflectivity. EM Technical Note.

Hughes Clarke J.E. (1994) - Towards remote seafloor classification using the angular response of acoustic backscatter: a case study from multiple overlapping GLORIA data. IEEE Journal of Oceanic Engineering, 19 (1): 112-127. doi:10.1109/48.289456

ICRAM CEDRE (2007) - Outcomes of the "Potentially Polluting Shipwrecks Workshop: technical, legal and financial perspectives”. 20 - 22 May 2007, Rome (Italy), pp. 12-14.

Jackson D.R., Winebrenner D.P., Ishimaru A. (1986) - Application of the composite roughness model to high-frequency bottom backscatter. J. Acoust. Soc. Am., 79: 1410-1422. doi:10.1121/1.393669

Kinsler E.L., Frey A.R., A.B. Coppens, J.V. Sanders. (2000) - Fundamentals of Acoustics. Fourth edition. John Wiley \& Sons, Inc., New York (USA).

Lurton X. (2002) - An Introduction to Underwater Acoustics. Principles and Applications. Springer-Praxis, Chichester (UK). 
MacDonald A.J., Collins C. (2008) - Taking Geocoder to Work. Making Geocoder part of your Daily Routine. Shallow Survey 2008.

Masetti G., Orsini F. (2009) - Environmental Risks Monitoring of Shipwrecks in Italian Seas. International Hydrographic Review, 11: 52-60.

Masetti G., Sacile R., Trucco A. (2010) - Caratterizzazione remota del fondale marino tramite analisi e mosaicatura del backscatter. Atti della $14^{\text {a }}$ Conferenza Nazionale ASITA (9 - 12 novembre 2010), Brescia (Italy).

Mayer L., Intro to Seafloor Classification. Lecture, 2011, Feb. 11, Durham (USA).

Mayer L., Fonseca L., Kraft B., Weber T., Trembanis A. (2007) - Remote Identification of Seafloor Properties in Denied Areas. Center for Coastal and Ocean Mapping Annual rept., Durham (NH).

Mulhearn P.J. (2000) - Modelling Acoustic Backscatter from Near-Normal Incidence Echosounders - Sensitivity Analysis of the Jackson Model. Defence Science \& Technology Organization, Melbourne (Australia).

Murphy L., Leary T., Williamson A. (1995) - Standardizing Seabed Classification Techniques. Sea Technology, 36: 15-19.

Parnum I.M., Gavrilov A.N., Siwabessy P.J.W., Duncan A.J. (2007) - Analysis of multibeam data for the purposes of seafloor classification. Proceedings of the $2^{\text {nd }}$ International Conference on Underwater Acoustic Measurements: Technologies \& Results, 25-29 June 2007, Heraklion (Greece).

PrestonJ.M.(2009)-Automatedacoustic seabed classification ofmultibeamimages of Stanton Banks. Applied Acoustics, 70 (10) :1277-1287. doi:10.1016/j.apacoust.2008.07.011

Preston J.M., Christney A.C., Beran L.S., Collins W.T. (2004) - Statistical seabed segmentation: from images and echoes to objective clustering. Proceedings of the $7^{\text {th }}$ European Conference of Underwater Acoustics, 813-816.

Stanton T.K. (1984) - Sonar estimates of seafloor microroughness. J. Acoust. Soc. Am., 75: 809-818. doi:10.1121/1.390590

Stockhausen J.H. (1963) - Scattering from the Volume of an Inhomogeneous Half-space. Rep. No. 63/9, Naval Research Establishment, Canada.

\section{Received 22/12/2010, accepted 11/05/2011}

